\title{
Preparation and Characterization of Two-Dimensional Ordered Arrays of Metallic Nanoparticles
}

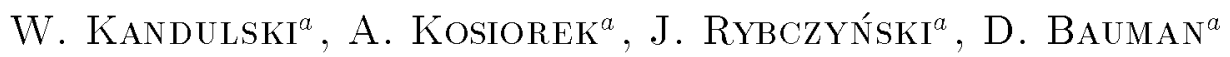 \\ AND M. GIERsig ${ }^{a, b}$ \\ ${ }^{a}$ Faculty of Technical Physics, Poznań University of Technology \\ Nieszawska 13a, 60-965 Poznań, Poland \\ ${ }^{b}$ Department of Nanoparticle Technology, Research Center CAESAR \\ Ludwig-Erhard-Allee 2, 53175 Bonn, Germany
}

(Received June 9, 2003; in final form August 6, 2003)

\begin{abstract}
A method of obtaining large-scale 2D arrays of ordered metallic nanoparticles is presented. The arrays were prepared using nanosphere lithography based on the self-assembly of latex particles on a water surface. Using $496 \mathrm{~nm}$ and $1040 \mathrm{~nm}$ diameter particles, it was possible to prepare arrays of over one $\mathrm{cm}^{2}$, with defect-free areas of over $50 \mu \mathrm{m}^{2}$. By the evaporation of nickel or gold, periodic metallic particle arrays were produced. Modification of the particle array morphology was also shown by using double-layered masks. Computer simulated mask models were used to reveal interesting morphologies obtainable by the application of multilayered masks.
\end{abstract}

PACS numbers: 81.07.-b

\section{Introduction}

Today's technology requires miniaturization. The smaller systems became, the faster and more economically they operate. However, it is both more difficult and more expensive to produce them. Nanotechnology searches for quick, reliable, and economically efficient methods of producing systems on the nanoscale. Nanosphere lithography promises to provide such a method of fabricating arrays of two-dimensional (2D) ordered nanoparticles based on self-assembly processes. One can use such 2D particle arrays as lithographic masks. This technique was 
originally proposed by Fischer and Zingsheim [1] in 1981 in their seminar work. The term of "naturally" assembled polystyrene (PS) latex nanospheres was then introduced. Later, in 1982, Deckman and Dunsmir [2] obtained a structure of ordered metallic nanoparticles with well-defined spacings through evaporation of a thin metallic overlayer onto nanosphere single layer (SL) templates. At that moment "natural" lithography (NL) became nanosphere lithography. To date, various techniques have been proposed for the fabrication of $2 \mathrm{D}$ arrays [3-7]. Despite many attempts, the results are still not satisfactory. The $2 \mathrm{D}$ templates obtained via the currently applied methods suffer from many imperfections manifesting themselves as loose packing, point defects, dislocations, and small areas of well organized structures. The best methods offer only a few $\mathrm{mm}^{2}$ of clean $2 \mathrm{D}$ ordered monolayer surfaces.

This paper presents an improvement upon the existing nanosphere lithography (NSL) techniques for creating nanoparticles monolayers. Some results were correlated with the data obtained from computer simulations.

\section{Experimental details}

\subsection{Materials}

Ultrapure water $\left(18.2 \mathrm{M} \Omega \mathrm{cm}^{-1}\right)$ was used directly from a Mili-Q water purifying system. Polystyrene nanospheres were purchased as a $10 \%$ wt. aqueous colloidal dispersion. The diameter of the nanoparticles was 496 and $1040 \mathrm{~nm}$ at a coefficient of variance $<0.3 \%$. The particles used had a hydrophobic surface and density of $1.05 \mathrm{~g} / \mathrm{cm}^{3}$.

Ethanol 98\%, acetone, toluene, and Triton X-100 surfactant were used. Samples were prepared using square, oriented (111), $n$-type silicon substrates $\left(5 \times 5 \mathrm{~mm}^{2}\right)$, with measured surface roughness $R_{\text {a }}$ parameter less than $1 \mathrm{~nm}$.

\subsection{Preparation of $2 D$ polystyrene nanospheres arrays}

PS masks were created as a self-assembly matrix on a liquid-air interface. A thin film of ethanol was spread over the water surface in a $10 \mathrm{~cm}$ large glass beaker. Next, the PS particle solution was slowly dropped at a constant speed onto the subphase surface using an Eppendorf pipette to form a floating layer. In order to improve its stability, Triton X-100 was added to water surface. An appropriate addition of this surfactant was experimentally determined depending on the layer size. Following this, a monolayer was lifted up onto a square Si substrate cleaned previously by ultrasonication in acetone and ethanol for 1 min each. During the distribution of PS colloid solution on top of the liquid a certain amount of particles sunk into the water volume. To remove this effect, the water containing the unwanted PS particles was locally removed using a little glass pipe. After cleaning, the monolayer was lifted off carefully and allowed to dry in air. The mask preparation time was ca. $20 \mathrm{~min}$. 


\subsection{Physical vapor deposition of $\mathrm{Ni}$ and $\mathrm{Au}$ overlayer}

Thin films of metals were deposited by electron beam evaporation in a BesTec UHV system under the pressure of $13 \times 10^{-7} \mathrm{~Pa}$. The thickness of deposited metal was controlled by a built-in quartz crystal microbalance (QCM) with a $2 \%$ measurement error. Samples were placed in a distance of $250 \mathrm{~mm}$ from effusive source. Depositing rate was $8-10 \AA / \mathrm{min}$. After deposition, the polystyrene particles were removed from the sample by ultrasonication in toluene to reveal metallic nanostructure on the substrate surface.

\subsection{Samples characterization}

The samples were scanned in air conditions using a Digital Instruments Nanoscope IIIa atomic force microscope (AFM) in contact mode. Pointprobe Silicon-AFM-Sensors cantilevers were used.

\section{Results and discussion}

\subsection{PS-templates characterization}

The final quality of metallic nanostructures depends directly on the quality of the PS matrix. Masks that are created by the method described above are distinguished by close packing order at large surface area $\left(c a .1 \mathrm{~cm}^{2}\right)$. The AFM image (see Fig. 1) reveals $496 \mathrm{~nm}$ PS particles which are arranged into a close

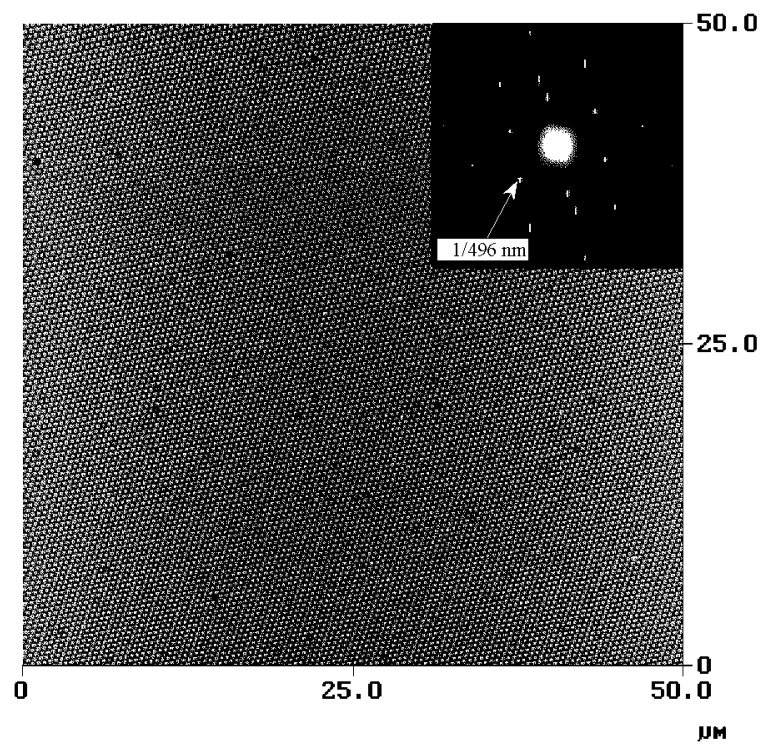

Fig. 1. AFM image of the $496 \mathrm{~nm}$ PS mask on Si substrate. FFT pattern is shown in the inset. 
packed monolayer. Nevertheless, a few point defects are observed. While a particle diameter deviation results in vacancies, the whole structure remains well ordered and no dislocations at regions larger than $50 \times 50 \mu \mathrm{m}^{2}$ are observed. Fast Fourier transform (FFT) confirms hep structure of the monolayer.

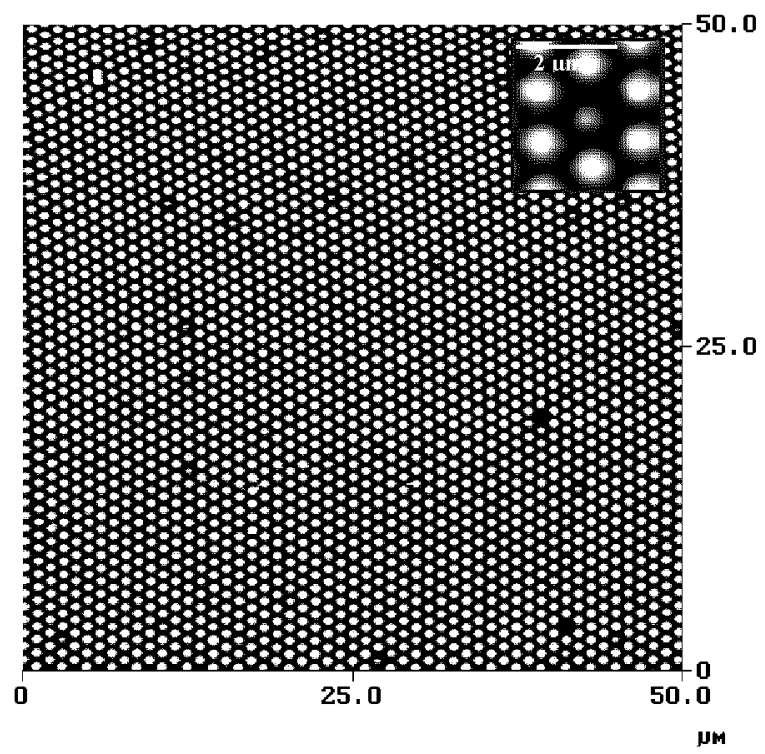

Fig. 2. AFM image of the $1040 \mathrm{~nm}$ PS mask on Si substrate. Inset shows structural inhomogeneity caused by smaller diameter particles.

Figure 2 presents the AFM image of the $1040 \mathrm{~nm}$ diameter PS sphere mask. Some point defects are visible, and are most likely caused by particles with smaller diameter (see inset in Fig. 2).

\subsection{Metallic particles characterization}

The deposition of different metals through the aforementioned masks gives periodically spaced metallic nanoparticles. The array obtained through the evaporation of a $10 \mathrm{~nm}$ thick $\mathrm{Ni}$ layer through the mask is shown in Fig. 3. The image remains in good correlation with latex mask quasi-triangular gaps. Two star-like objects in the image are caused by vacancies in the PS mask.

Figure 4a shows an enlarged fragment of the structure presented in Fig. 3 with the measured AFM profile. The image reveals that the edges of the evaporated metallic particles are not perfectly sharp. This is a consequence of non-point effusing source corresponding to the sample size. Morphological analysis and the profile measurement are presented in Fig. 4b. The measured height of the examined particle is approximately $11.5 \mathrm{~nm}$, which is in good agreement with the value of $10 \mathrm{~nm}$ set during the evaporation process considering the surface roughness, 


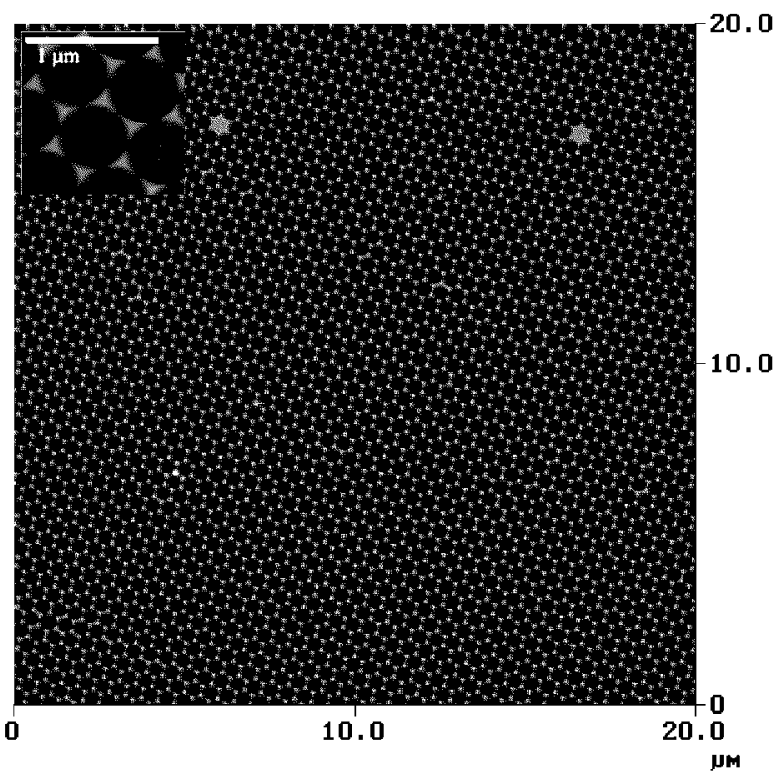

Fig. 3. AFM image of Ni particles evaporated through the $496 \mathrm{~nm}$ PS mask.
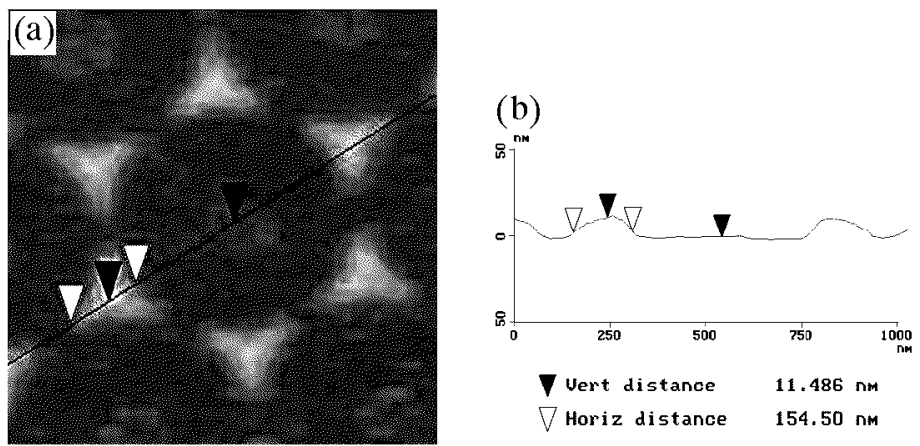

Fig. 4. Morphological analysis with profile measurement (b) of selected Ni particles evaporated through the $496 \mathrm{~nm}$ PS mask (a). The nickel layer deposited onto the mask was $10 \mathrm{~nm}$ thick.

$R_{\mathrm{a}}$, equal to $1 \mathrm{~nm}$ and $0.2 \mathrm{~nm}$ evaporation thickness error. The measured in-plane height of the quasi-triangular Ni shape was $154.5 \mathrm{~nm}$.

\subsection{Simulation and characterization of metallic structures based on multilayered masks}

Through the deposition of a second PS layer onto a previously covered sample surface it is possible to create more complex morphologies of metallic particle arrays. Structures based on double masks vary due to the relative orientation and 
shift of each PS monolayer. The main difficulty during preparation of multilayered structures involves precise control of the revolution angle between the two layers. This problem can be solved by determining the orientation of every layer during the preparation process, through the analysis of the diffraction pattern obtained upon reflection of a laser beam incident on the monolayer. However, such angle control is reliable only if the layer is a monocrystal and the light wavelength is comparable to the particle size.
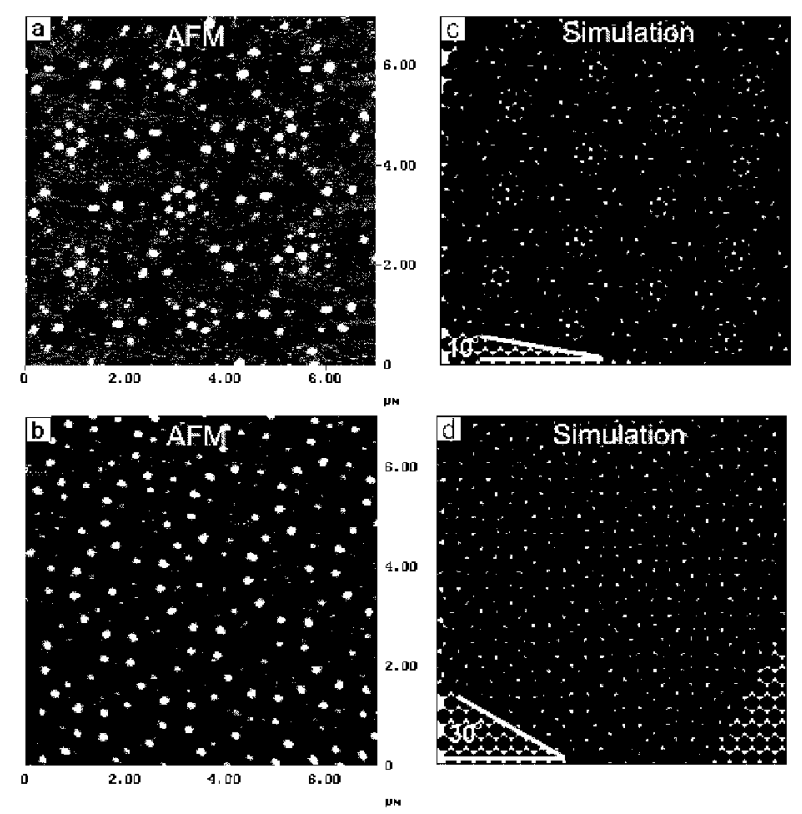

Fig. 5. Nickel nanostructures (a,b) evaporated through a double $496 \mathrm{~nm}$ PS layer. The alignment angles between the first and second PS layers were $10^{\circ}$ (c) and $30^{\circ}$ (d).

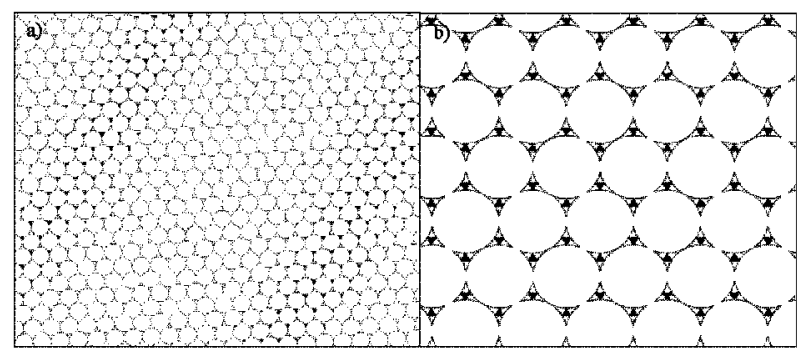

Fig. 6. Computer simulations of various possible structures. (a) Projection through a threefold layered mask with alignment modifications, (b) projection through a double layered mask with sphere size modifications. 
Figures $5 \mathrm{a}$ and $\mathrm{b}$ show structures obtained by the evaporation of Ni through double PS masks, fabricated without revolution angle control. The angle between PS monolayers was obtained through simple computer simulation (Figs. $5 \mathrm{c}$ and d) correlated with the original AFM images. Computer simulations were performed in image editing program - Adobe Photoshop by rotating virtual layers of hcp-ordered spheres. By comparing original images with simulated images, the revolution angle was found. A wide variety of structures can be produced by simple changing the alignment parameters between layers and by using different PS particle sizes.

Figure 6 presents simulations of such multilayered structures. Projection through three layers deposited one after another is shown in Fig. 6a, where the first two PS sphere layers form an hep structure and the third layer is deposited at the angle of $58^{\circ}$ to the previous two ones. Figure $6 \mathrm{~b}$ shows a projection through a double layered mask, where one layer is built from spheres twice bigger than those in the other one.

\subsection{Summary}

It was shown how relatively large, ordered arrays of metallic particles were created using nanosphere lithography. Improvements on other NSL methods were realized by increasing the area over which the PS monolayer is homogeneously ordered up to ca. $1 \mathrm{~cm}^{2}$ and by decreasing the number of structural defects. AFM images showing dislocation-free $50 \times 50 \mu \mathrm{m}^{2}$ areas of PS sphere mask were presented. Control over the morphology of metallic structures was possible through adjustment of the PS particle size and of the metal evaporation parameters. The method is both inexpensive and time efficient. The possibility of fabricating multilayered masks promises other future applications. By evaporating different metals in one process, it is possible to prepare composite structures [8]. In conclusion, NSL seems to become one of the most promising lithography methods.

\section{Acknowledgments}

We gratefully acknowledge the financial support from the Research Project No. 5 P03B 10021 coordinated by the State Committee for Scientific Research (Poland) and from European Union under contract No. HPRN-CT-1999-00150.

\section{References}

[1] U.C. Fischer, H.P Zingsheim, J. Vac. Sci. Technol. 19, 881 (1981).

[2] H.W. Deckman, J.H. Dunsmir, Appl. Phys. Lett. 41, 377 (1982).

[3] M.P. Tessier, O.D. Velev, A.T. Kalambur, A.M. Lenhoff, J.F. Rabolt, E.W. Kaler, Adv. Mater. 13, 396 (2001).

[4] C.R. Rossi, M.X. Tan, N.S. Lewis, Appl. Phys. Lett. 77, 2698 (2000). 
[5] J.C. Hulteen, R.P. Van Duyne, J. Vac. Sci. Technol. 13, 1553 (1995).

[6] P.N. Bartlett, P.R. Birkin, M.A. Ghanem, Chem. Commun. 17, 1671 (2000).

[7] B. Gates, D. Quin, Y. Xia, Adv. Mater. 11, 466 (1999).

[8] J. Rybczyński, M. Hilgendorff, M. Giersig, in: NATO Science Series II, Dynamic Interactions in Quantum Dot Systems, Eds. L.M. Liz-Marzán, M. Giersig, Kluwer Academic Publishers, Dordrecht 2003, p. 163. 